\title{
Additional thoughts on teaching and prescribing MAOls
}

\author{
S. Shalom Feinberg*
}

Department of Psychiatry and Behavioral Sciences, Albert Einstein College of Medicine/Montefiore Medical Center, Bronx,

New York, United States

Received 7 March 2017; Accepted 9 March 2017; First published online 18 May 2017

Key words: MAOIs, monoamine oxidase inhibitors, serotonin syndrome, hypertensive reaction, dietary tyramine.

\section{To THE EDITOR:}

I would like to share some thoughts about Dr. Gilman's recent editorial entitled "Much ado about nothing': monamine oxidation inhibitors, drug interactions, and dietary tyramine."1 Unquestionably an international expert on the subject, Dr. Gilman continues to provide a great service in championing the use of MAOIs for refractory depressive illness. His research has been immeasurably helpful to those of us who prescribe and teach about the use of MAOIs.

Parallel with Dr. Gillman's paper, the frequency of hypertensive reactions in my 30 plus years of prescribing MAOIs has been quite low. But I have found there to be more variability in real life than he reports from the literature. ${ }^{2}$ For instance, I have seen several hypertensive reactions of varied intensity over the years coming from a variety of sources, even when the food or quantity of food ingested was reportedly low in tyramine. (I investigated each case to ascertain the possible sources of tyramine.) I have had two patients whose hypertensive reaction required brief hospitalizations. Fortunately, the outcomes in all these cases were medically favorable, but not all the patients continued with MAOI treatment due to the stress connected to their reactions. In supervising trainees about MAOIs, I try to prepare them for these realities and, I daresay, the risks. Because of these potential risks, appropriate consent is mandatory.

A primary cause of hypertensive reactions in my practice in recent years has been the ingestion of foods that unbeknownst to the patient had spoiled. This point was not stated in Dr. Gilman's editorial, but concrete patient education around these issues needs to be emphasized during the training process. For example, I emphasize to patients that, if in doubt about a food's freshness, throw it out, send it back (if eating in a

\footnotetext{
* Address for correspondence: S. Shalom Feinberg, 108-28 68th Drive, Forest Hills, New York 11375, USA.

(Email: shalomf@aol.com)
}

restaurant), or (tongue firmly in cheek) give it to your spouse.

Also in terms of the training process, I think that careful patient selection is a particularly vital issue in prescribing MAOIs, a point not mentioned by Dr. Gilman. It is critical that the clinician evaluate the patient's ability to understand the implications of using these medicines and their ability to reach out if there are any questions regarding them. ${ }^{3}$ For example, I am aware of the recent case of a young adult who is presently comatose after apparently having ingested escitalopram while having been recently prescribed phenelzine. I do not advocate frightening patients unnecessarily, but they must be mature enough to participate in responsible care. Similarly, if possible, having family members involved is useful for enhancing responsible care. ${ }^{3}$

It is essential that psychiatric residents and all new prescribers of MAOIs receive proper education and supervision in the early stages of their prescribing these potentially lifesaving medicines.

In summary, I certainly agree with much of the substance of Dr. Gillman's editorial, but I'd be more comfortable with "Much ado about something"-a very important something, something worth learning.

\section{Disclosures}

Shalom Feinberg hereby declares that he has nothing to disclose.

\section{REFERENCES:}

1. Gillman K. "Much ado about nothing": monamine oxidation inhibitors, drug interactions, and dietary tyramine. CNS Spectr. 2017; 2: 1-3; Epub ahead of print.

2. Cole JO, Bodkin AJ. MAO inhibitors: an option worth trying in treatment-resistant cases. Curr Psychiatry. 2002; 1(6): 40-47.

3. Fawcett J. Why aren't MAOIs used more often? J Clin Psychiatry. 2009; 70(1): 139-140. 